\title{
Editorial on trigonocephaly
}

\author{
Saffet Mutluer
}

Received: 5 July 2013 / Accepted: 17 July 2013 / Published online: 9 August 2013

(C) Springer-Verlag Berlin Heidelberg 2013

Trigonocephaly, which is called as "metopic suture synostosis", is a single-suture synostosis that is commonly seen. However, in recently published manuscripts, the incidence is slowly increasing. In the series regarding epidemiology, it appears that it is catching up with the sagittal suture synostosis, which was previously the most frequently seen craniosynostosis type. It is difficult to explain the cause of the increase of the trigonocephaly incidence. I would like to underline a couple of interesting points: Firstly, we do not know the natural history of trigonocephaly. Secondly, when we go through the literature, we do not find anything belonging to the adult trigonocephaly, except one to two cases that were published before. Yet, there are some adult patients with other type craniosynostosis cases were published. During my personal communication, a few pediatric neurosurgeons have mentioned that they have seldom seen adult trigonocephaly patients, but they did not document the cases. We are yet to know if some trigonocephaly cases recover without treatment or they are well because all of them were operated on that is why we do not $t$ see any adult patients.

Radiologically or pathologically, we observe a sharp angle and keel shape on the fronto-orbital area. Theoretically, there is a structural abnormality in the skull base. At the essential of the surgical treatment, the fronto-orbital angle is straightened. Then, the frontal bones are shaped with various surgical techniques. Many surgical techniques have been published; however, in all of them, the anterior skull base is reconstructed first. So, how does the skull base take normal shape as a result of the endoscope-assisted metopic suture removal, which has become widespread lately? We do not know much about this.

Mostly positive cosmetic outcomes are received as a result of surgical treatment. There is insufficient data regarding secondary synostosis and rate of reoperations. Trigonocephaly is generally sporadic. However, it can be seen along with some syndromes. It is usually found with FGFR 1 anomalies. Unfortunately, there is insufficient knowledge on the genes that affect the formation of the base of the skull and cause trigonocephaly.

Many studies show the intracranial pressure (ICP) increase even in simple suture synostosis. However, in some trigonocephaly cases, an increased ICP has been published. The exact cause is also not well understood. The compression of the frontal lobes, by the narrowing of the frontal area, causes neurobehavioral and neurocognitive disorders. In some published series, it was reported that these were recovered after the surgical intervention. The extent to how much these have improved and the effect of the timing of the surgeries are not precisely known.

In this focus issue, we search for the answers of some of the questions I have mentioned above. However, I believe that we need to do numerous researches to be able to answer all of the questions.

\footnotetext{
S. Mutluer $(\bowtie)$

Neurosurgery/Pediatric Neurosurgery, Private Gazi Hospital, Izmir, Turkey

e-mail: saffetmutluer@gmail.com
} 